\title{
Variations
}

Variations Revue internationale de théorie critique

$16 \mid 2012$

\section{Tahrir is here !}

\section{Encre rouge}

\section{Slavoj Žižek}

Traducteur : Christel Pesme

\section{CpenEdition}

\section{Journals}

\section{Édition électronique}

URL : http://journals.openedition.org/variations/194

DOI : 10.4000/variations. 194

ISSN : 1968-3960

Éditeur

Les amis de Variations

Édition imprimée

Date de publication : 16 janvier 2012

Référence électronique

Slavoj Žižek, «Encre rouge », Variations [En ligne], 16 | 2012, mis en ligne le 20 février 2012, consulté le 20 avril 2019. URL : http://journals.openedition.org/variations/194 ; DOI : 10.4000/variations.194

Ce document a été généré automatiquement le 20 avril 2019.

Les ami•e•s de Variations 


\title{
Encre rouge
}

\author{
Slavoj Žižek
}

Traduction : Christel Pesme

\section{NOTE DE L'ÉDITEUR}

Prise de parole lors d'une assemblée Occupy Wall Street en octobre 2011 à Madison Square

Première publication sur www.theoriecritique.com, « Tahrir is here ! ", 16 janvier 2012, pp. $14-17$

1 Ne tombez pas amoureux-ses de vous-mêmes, de ce chouette moment que nous passons maintenant. Les carnavals sont souvent de pacotille et le vrai test de ce qu'ils valent est ce qui en reste le lendemain, comment la vie quotidienne en sort transformée. Aimez le travail dur et patient - nous ne sommes que le commencement, pas la fin. Notre message est simple : le tabou est brisé - nous ne vivons pas dans le meilleur des mondes - et il nous est permis, nous y sommes même obligé-es, de penser les alternatives.

2 Le chemin à parcourir est long et nous serons bientôt confronté-e-s aux questions vraiment difficiles - questions non pas à propos de ce que nous ne voulons pas, mais plutôt autour de ce que nous voulons vraiment. Quelle organisation sociale peut remplacer le capitalisme existant? Avec quels types de nouveaux dirigeants? Etant entendu que les alternatives du 20e siècle ont échoué.

3 Il ne sert donc à rien de blâmer les gens et leurs attitudes: le problème n'est ni la corruption ni l'appât du gain, le problème est le système qui pousse à être corrompu-e. La solution n'est pas « Main Street contre Wall Street » (la voix du peuple contre la voix des banquiers) mais de changer ce système dans lequel le peuple ne peut exister sans les banquiers.

4 Méfions-nous de nos ennemis mais aussi de nos faux-amis - de ceux qui tout en prétendant nous soutenir s'acharnent déjà à affaiblir notre contestation. De la même façon que nous avons déjà du café sans caféine ou de la bière sans alcool, ils essaient de 
nous réduire à une inoffensive contestation morale. Mais la raison pour laquelle nous sommes ici est que nous en avons marre de ce monde ou recycler nos canettes de Cocacola, donner trois francs 6 sous pour les pauvres ou acheter des cappuccinos à Starbucks parce que X\% du prix servirait à régler les problèmes du tiers-monde, est suffisant pour nous donner bonne conscience. Apres la délocalisation du travail et de la torture, après que les agences maritales aient aussi commencé à délocaliser nos potentielles rencontres amoureuses, nous nous apercevons que nous avons aussi permis que notre engagement politique soit lui aussi sous-traité - nous voulons nous réapproprier tout ça.

Ils vont nous dire que nous sommes anti-américains. Mais quand les chrétiens fondamentalistes nous disent que l'Amérique est une nation chrétienne, il faut se souvenir de ce que la chrétienté est : le Saint Esprit, la communauté libre et égalitaire des croyants unis dans l'amour. Ici, nous sommes le Saint Esprit alors que c'est à Wall Street que se trouvent les païens vénérant de fausses idoles.

6 Ils vont nous dire que nous sommes violents, que notre langage même est violent: occupation, etc., etc. Oui, nous sommes violents, mais seulement au sens où le Mahatma Gandhi était violent. Nous sommes violents parce que nous voulons arrêter le cours des choses. Mais quelle est cette violence purement symbolique si on la compare à la violence requise au maintien du fonctionnement sans accroc du système capitaliste global ?

7 Ils nous appellent des losers - mais les vrais perdants ne sont-ils pas ceux qui à Wall Street se sont fait renflouer de centaines de milliards de notre argent? On vous traite de socialistes - mais aux US, le socialisme existe déjà mais seulement pour les riches. Ils vont vous dire que vous ne respectez pas la propriété privée - mais ce sont les spéculations de Wall Street qui ont conduit au krach de 2008 et qui ont détruit plus de propriétés privées durement constituées que si nous voulions le faire ici jours et nuits - pensez juste aux milliers de maisons saisies par les banques.

Nous ne sommes pas communistes, si communiste se réfère au système qui s'est justement effondré dans les années 90 - et souvenez-vous que ceux de ces communistes qui sont encore au pouvoir appliquent le capitalisme le plus sauvage (en Chine). Le succès du capitalisme rouge chinois est le signe inquiétant que le mariage entre capitalisme et démocratie est au bord du divorce. Le seul sens dans lequel nous sommes Communistes est que nous prenons soin des biens communs - ceux de la nature, de la connaissance que le système met en danger.

9 Ils vont vous dire que vous êtes des rêveurs, mais les vrais rêveurs sont ceux qui pensent que les choses peuvent continuer indéfiniment, telles quelles, qu'il suffit juste d'en maquiller un peu l'apparence. Nous ne sommes pas des rêveurs-euses, nous nous réveillons d'un rêve qui tournait au cauchemar. Nous ne détruisons rien, nous ne sommes que les simples témoins de comment le système est en train, petit à petit, de s'autodétruire. Nous connaissons toutes et tous la fameuse scène de dessin animé : le chat est au bord du précipice mais il continue de marcher ignorant du fait qu'il n'y a plus de terre ferme sous ses pieds : il ne tombe qu'à partir du moment où, jetant un œil en bas, il prend conscience de l'abysse. Ce que nous faisons est juste de rappeler à ceux qui sont au pouvoir de regarder en bas...

10 Alors, le changement est-il vraiment possible? De nos jours, le possible et l'impossible s'organisent de façon quelque peu étrange. Dans les domaines des libertés individuelles et des technologies scientifiques, l'impossible devient de plus en plus possible (d'après ce que l'on nous en dit) : « rien n'est impossible», on peut profiter du sexe dans toutes ses 
versions perverses; des archives entières de musiques, de films et de séries télé sont disponibles au téléchargement; traverser l'espace est possible pour tout un chacun (ayant de l'argent...) ; il est possible d'augmenter nos capacités physiques et psychiques grâce à des interventions au niveau du génome; tout semble devenu possible jusqu'au rêve techno-gnostique d'arriver à l'immortalité en transformant notre identité en un programme d'ordinateur.

De l'autre coté, dans le domaine des relations sociales et économiques, nous sommes bombardé-e-s sans relâche d'un «tu ne peux pas » : «tu ne peux pas t'engager dans des actions politiques collectives (qui nécessairement aboutissent à la terreur totalitaire), ou te cramponner au vieil Etat-providence (il te rend non compétitif et conduit aux crises économiques), ou t'extraire du marché financier global, etc, etc.» Quand les mesures d'austérité sont imposées, on nous répète encore et encore qu'on peut tout simplement pas faire autrement. Peut-être le moment est-il venu d'inverser les coordonnées de ce qui est possible et de ce qui est impossible ; peut-être ne pouvons-nous pas devenir immortels mais pouvons-nous vivre dans plus de solidarité et de sécurité sociale.

Mi-avril, les médias rapportaient que le gouvernement chinois avait interdit de diffuser à la télé comme au cinéma tout film abordant les thèmes en lien avec le voyage dans le temps ou l'histoire parallèle, selon l'argument que de telles histoires introduisent de la frivolité au contenu historique sérieux - même l'échappée fictionnelle dans une réalité parallèle est considérée comme trop dangereuse. Nous, dans l'Ouest libéral, nous n'avons pas besoin d'interdits aussi explicites : l'idéologie exerce assez de pouvoir matériel pour prévenir tout risque de prendre un minimum au sérieux de telles histoires parallèles. Comme le montre la pléthore de films apocalyptiques, il nous est plus facile d'imaginer la fin du monde que celle du capitalisme.

Dans une ancienne plaisanterie de la défunte RDA, un travailleur Allemand trouve un boulot en Sibérie. Conscient que son courrier sera ouvert et censuré, il dit à ses amis : « Mettons-nous d'accord sur un code : si la lettre que vous recevez est écrite à l'encre bleue ordinaire, cela signifie que son contenu est vrai ; si elle est écrite en rouge, son contenu est faux. » Après un mois, ses amis reçoivent la première lettre, écrite en bleu : « Tout est fantastique ici : les magasins sont remplis, la nourriture est abondante, les appartements sont grands et bien chauffés, les cinémas jouent des films de l'Ouest, il y a plein de jolies filles prêtes à une aventure - la seule chose qui manque, c'est de l'encre rouge. »

N'est-ce pas la situation dans laquelle nous sommes? Nous avons toutes les libertés voulues - la seule chose qui manque est «l'encre rouge » : nous nous «sentons libres" parce le langage même pour penser notre manque de liberté nous fait défaut. Le manque d'encre rouge signifie qu'aujourd'hui, tous les mots les plus couramment employés pour désigner le conflit actuel - "guerre à la terreur ", « démocratie et liberté », «droits de l'homme ", etc. - sont des termes falsifiés qui mystifient notre perception de la situation au lieu de nous permettre de la penser.

Vous, ici, vous nous fournissez à toutes et à tous de l'encre rouge. 


\section{AUTEURS}

SLAVOJ ŽIŽEK

Philosophe, psychanalyste, et acteur des mouvements alternatifs slovènes 